\title{
How to prevent intra-operatory capsular blockage? a new surgical approach: the anti-blockage technique
}

\begin{abstract}
Aim: To describe a novel technique that prevents intraoperative capsular blockage in phacoemulsification surgery.

Introduction: Cataract surgery by phacoemulsification is the technique of choice for most cataract surgeons worldwide. This technique can have complications at any of its stages. The frequency of the presentation of intraoperative complications varies according to several factors. These include the surgeon's experience, the type of cataract and whether suitable instruments are used. The complications can potentially be prevented if good techniques are performed. Capsular blockage syndrome (CBS) is a recognised complication that can occur during the phacoemulsification or after surgery. In this article we will focus on a technique used to prevent CBS which we have called 'The Antiblockage Technique'.
\end{abstract}

Methods: We describe a novel technique that uses the Escaf Ultra Chop blade ${ }^{\mathbf{T M}}$ (EUC) to prevent intraoperative capsular blockage in a group of 669 eyes of patients. The study was performed at the Clinica Oftalmologica del Caribe in the city of Barranquilla, Colombia from January to June 2011. All the patients were diagnosed with cataracts that were classified according to LOCS III. These patients underwent phacoemulsification using the EUC ${ }^{\mathbf{T M}}$ and the antiblockage technique with IOL implantation. The surgeons participating in the study included experienced cataract surgeons and their trainees.

Results: All of the patients had a satisfactory anatomical outcome with no CBS reported.

Conclusion: This novel technique helps cataract surgeons prevent the intraoperative CBS and its complications. It is especially useful for surgeons in training as it is easy to master. This technique can be performed safely with the EUCTM
Volume 7 Issue 2 - 2017

Luis Jose Escaf, Luz Marina Melo, Juana Londoño, Claudia Quijano, Luis Carlos Escaf Department of ophthalmology, Clinica Oftalmologica del Caribe, Colombia

Correspondence: Luis Jose Escaf, Especialista en catarata, cornea y cirugia refractive, Director Cientifico, Clinica Oftalmologica del Caribe, Torre Medica del Mar, Sala Azul, Calle 85 N 50-37, Barranquilla, Colombia, Email escaff@gmail.com

Received: June 10,2017| Published: July 10, 2017
Abbreviations: CBS, capsular blockage syndrome; EUC, escaf ultra chop blade ${ }^{\mathbf{T M}}$, PCR, posterior capsular rupture; OVD, ophthalmic viscoelastic device; IOL, intraocular lens

\section{Introduction}

Phacoemulsification is the technique of choice for most cataract surgeons worldwide. This technique can have complications at any of its stages, including intra or post-operative stages. The frequency of the presentation of intraoperative complications varies according to the surgeon's experience, ${ }^{1}$ the type of cataract and whether suitable instruments are used. ${ }^{2}$ Capsular blockage syndrome (CBS) is one of the recognised intra-operative complications that can occur during the phacoemulsification.

\section{Pathophysiology of the intraoperative capsular blockage}

The intraoperative CBS is a condition characterized by accumulation of fluid between the anterior surface of the posterior capsule and the epinucleus. This can happen during the hydrodissection manoeuvre. If the hydrodissection is performed abruptly, the injected fluid displaces the nucleus forward, blocking the capsulorhexis. Research has shown that the hydrodissection manoeuvre can cause a rise in the intraocular pressure (up to $223 \mathrm{~mm} / \mathrm{Hg}$ ) during the phacoemulsification ${ }^{3,4}$ (Table 1). In addition, the rapid increase in volume within the capsule produces a sudden posterior capsular distension that could lead to an inadvertent posterior capsular rupture (PCR) and a luxation of the nucleus to the vitreous cavity. Signs that indicate that CBS has happened are: ophthalmic viscoelastic device (OVD) loss through the main wound, iris herniation with shallowing of the anterior chamber and a rise in the ocular tone (Figure 1). If the PCR is not suspected and surgery is continued, the nucleus or fragments can drop to the vitreous cavity and this will complicate the surgery further.

Table I N=57; Epidemiological distribution of the pathological fractures, traumatic fractures, and nonunion

\begin{tabular}{ll}
\hline Surgical Step & IOP $(\mathrm{mm} / \mathrm{Hg})$ \\
\hline Viscoelastic injection & 163 \\
Irrigation/Aspiration & 82 \\
Intraocular lens implantation & 121 \\
Hydrodissection & $223^{*}$ \\
\hline
\end{tabular}

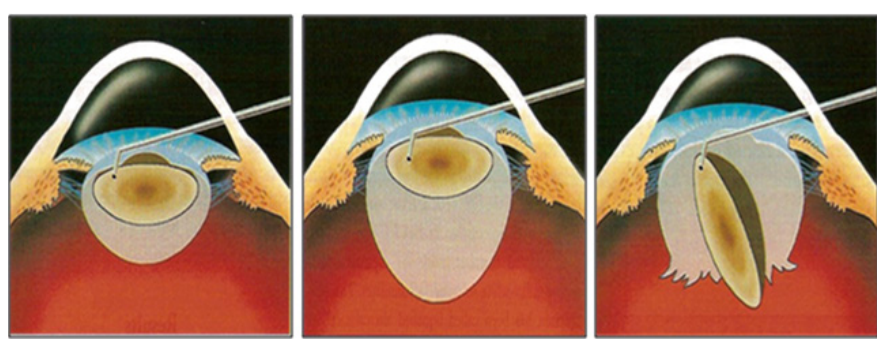

Figure I CBS is a complication of cataract surgery where the capsular bag accumulates fluid between the lens and the posterior capsule after hydrodisection is performed. This trapped fluid can cause distension and subsequent rupture of the posterior capsule and luxation of the lens into the vitreous cavity. Original from Dr Luis J Escaf 
CBS can also happen after an intraocular lens (IOL) implantation. The main reason for its occurrence is due to a circumferential adherence between the anterior capsular opening and the optic plate of the IOL. It is characterized by a capsular hyperexpansion, anterior displacement of the IOL, reduction of the anterior chamber depth and myopic shift after the phacoemulsification with IOL implantation.

Davison in 1990, was the first to describe the CBS. ${ }^{5}$ Miyake classified it according to the time of onset: ${ }^{6}$

1. Intraoperative: $\mathrm{CBS}$ at the time of the hydrodissection

2. Early postoperative: Original CBS - presenting within the first few weeks following surgery

3. Late postoperative: CBS with liquefied after cataract - months to years following surgery

Within the differential diagnosis we can find choroidal detachment, expulsive haemorrhage and misdirection syndrome. There have been different treatment options described before according to the clinical scenario. ${ }^{7-9}$ In this manuscript we will describe a new surgical technique that we have called 'The Antiblockage Technique'. This prevents the intra-operatory CBS during hydrodissection in the phacoemulsification surgery.

\section{Objective}

Toprevent the rupture of the posterior capsule in phacoemulsification during the hydrodissection. The technique will be described using the Escaf Ultra Chop ${ }^{\mathbf{T M}}$ (EUC) - (designed by Dr Luis Escaf).

\section{Materials and methods}

A prospective study on a consecutive 669 eyes of patients (age range between 50-75) that were diagnosed with cataract and underwent phacoemulsification in a period of 6 months was completed. The site of the study was the Clinica Oftalmologica del Caribe in the city of Barranquilla, Colombia, and the study was conducted from January to June 2011. All the cataracts were classified according to LOCS III. Phacoemulsification was performed using the EUC'M and the antiblockage technique and all the eyes had an IOL implantation. The surgeons participating included experts and trainee cataract surgeons.

\section{Inclusion criteria}

1. Posterior subcapsular cataract

2. Polar cataract

3. Voluminous nucleus

4. Shallow anterior chamber

5. Medium mydriasis

6. IFIS patients

7. Small capsulorhexis

\section{Surgical technique}

1. Topical anesthesia

2. Auto sealant incision

3. Continuous circular capsulorhexis (CCC) 5.0-5.5 mm of diameter
4. First Grove with EUCTM and nucleus division with Escaf divisor ${ }^{\mathbf{T M}}$

5. Hydrodissection and rotation of the nucleus

6. Multiple division of the nucleus in more fragments with EUC ${ }^{\mathbf{T M}}$

7. Phacoemulsification (Infiniti machine and Escaf ${ }^{\mathbf{M M}}$ manipulator)

8. IOL implantation

\section{Antiblockage manoeuvre}

The antiblockage technique, which utilizes the EUCTM in our group of patients, was designed to prevent intraoperative CBS. After topical anaesthesia is instilled, a corneal self-sealing incision is performed. A CCC is created to then make a deep cut in the nucleus with the $\operatorname{EUC}^{\mathbf{T M}}{ }^{10}$ (Figure 2A). Immediately after the central nucleus is cut, a complete nucleus fracture with a nucleus divisor (K3-2387) or a mechanical pre-chopper ${ }^{11}$ (Escaf design) is performed. This allows us to have a communication between the anterior and the posterior surface of the cataract (Figure 2B). When two halves of the cataract is obtained, a gentle hydrodissection is performed. While the saline solution is injected to perform the hydrodissection, the injected fluid can be seen circulating through the space created between the two fragments of the cataract going upwards into the anterior chamber (Figure 3).

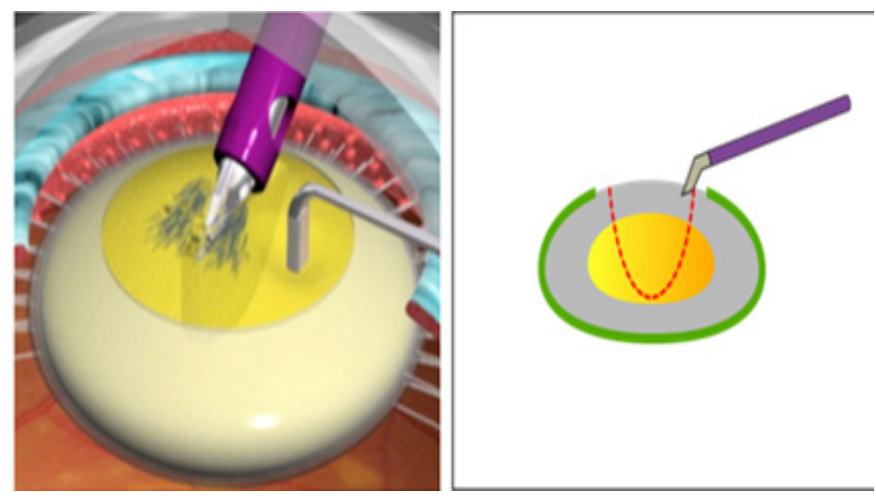

Figure 2 Antiblockage technique using the Escaf Ultra Chop.

(A) After CCC a central groove in the nucleousis created with the EUC. Original from Dr Luis J Escaf

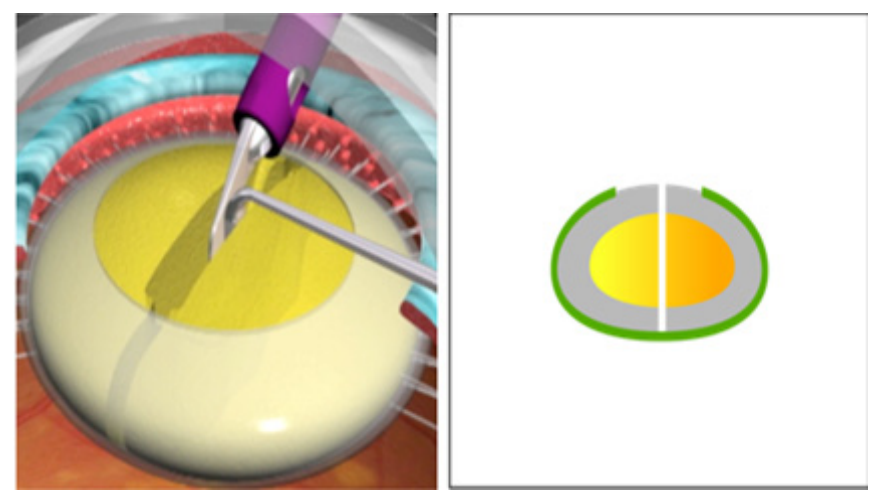

Figure 2 Antiblockage technique using the Escaf Ultra Chop.

(B) The Groove is split in to two halves, helped with a second instrument. The division of the nucleus allows a pathway from the anterior capsule to the posterior capsule where the fluid can run freely. Original from Dr Luis J Escaf 


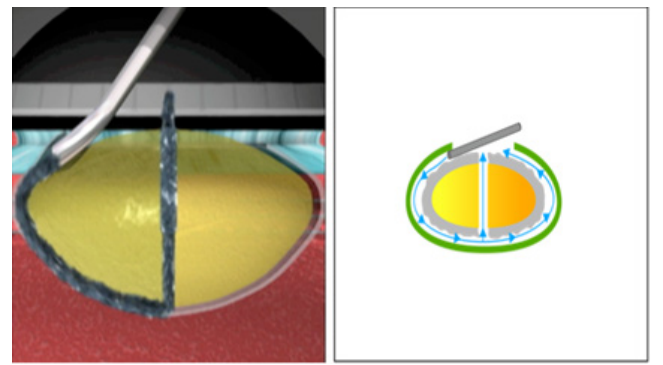

Figure 3 Hydrodisection.

Hydrodisection: The fluid runs between the two halves releasing the tensión over the posterior capsule and avoiding blockage of the CCC. Original from Dr Luis J Escaf

This new path eases the fluid circulation and avoids the accumulation between the back of the lens and the posterior capsule, thus preventing distension of the capsular bag and consequent rupture. The above described manoeuvre can be confirmed when vision blue is injected. It can be seen clearly how this fluid goes upwards through the two halves of the cataract when the hydrodissection is done. After this, the two halves are rotated and the fracture of the cataract with the EUC'M can be completed. The potential complications of this technique are that we could cut the anterior capsulorhexis and that the nucleus cannot be completely fractured after the first attempt. None of the previous complications mentioned could eventually stop us for continuing the surgery, as they can both be managed immediately after they happen.

\section{Results}

From the 669 eyes where we performed antiblockage technique, none of them had intraoperatory capsular blockage. The characteristics of the surgery and type of cataract can be seen in Table 2 .

Table 2 Number of cases that did not present capsular blockage using the antiblockage technique

\begin{tabular}{lll}
\hline Characteristic of surgery/type of cataract & Number of cases & Blockage \\
\hline Posterior subcapsular cataract & 80 & No \\
Polar cataract & 68 & No \\
Medium midriasis & 31 & No \\
Shallow anterior chamber & 52 & No \\
IFIS & 8 & No \\
Voluminous cataract & 50 & No \\
Small CCC & 80 & No \\
Fellows in training & 300 & No \\
Total & 669 & 0 \\
\hline
\end{tabular}

Recommended scenarios when to use the antiblockage technique

1. Shallow anterior chamber

2. Voluminous cataract

3. Short eyes

4. Medium mydriasis

5. Posterior Polar Cataract

6. Medium capsulorhexis

7. IFIS

8. Posterior subcapsular cataract

9. Pseudoexfoliative cataract

10. Surgeons in training

11. Others considered by the surgeon

\section{Conclusion}

We have presented a new reproducible surgical technique that avoids the intra-operatory capsular blockage and prevents a capsular rupture when the hydrodissection is performed. From 669 phacoemulsification cases where the antiblockage technique was performed, no cases presented a blockage capsule and so it was proven to be safe and easy to perform. This novel technique helps cataract surgeons prevent the intraoperative capsular blockage and its complications, and it is especially useful for surgeons in training. This technique can be performed safely with the EUC.

\section{Acknowledgments}

None.

\section{Conflicts of interest}

The authors declare no conflicts of interest.

\section{References}

1. Mangan MS, Atalay E, Arici C, et al. Comparison of Different Types of Complications in the Phacoemulsification Surgery Learning Curve According to Number of Operations Performed. Turk J Ophthalmol. 2016;46(1):7-10.

2. Roberts TV, Sutton G, Lawless MA, et al. Capsular block syndrome associated with fem to second laser-assisted cataract surgery. $J$ Cataract Refract Surg. 2011;37(11):2068-2070.

3. Vasavada. Real-Time-Intraocular Pressure (IOP) Measurement during Phacoemulsification: Randomized clinical trial. AJOC. 2010;4-6.

4. Howard Fine. Intraocular pressure during phacoemulsification. J Cataract Refract Surg. 2006:132. 
5. Davison JA. Capsular bag distension after endo phacoemulsification and posterior chamber intraocular lens implantation. J Cataract Refract Surg. 1990;16(1):99-108.

6. Miyake K, Ota I, Ichihashi S, et al. New clasification of capsular block syndrome. J Cataract Refract Surg. 1998;24(9):1230-1234.

7. Durak I, Ozbek Z, Ferliel ST, et al. Early postoperative capsular block syndrome. J Cataract Refract Surg. 2001;27(4):555-559.

8. Yepez JB, Cedeño de Yepez J, Arevalo JF. Intraoperative peripheral anterior capsulotomy to prevent early postoperative capsular block syndrome. J Cataract Refract Surg. 2004;30(9):1840-1842.
9. Vélez M, Velásquez LF, Rojas S, et al. Capsular block syndrome: a case report and literature review. Clin Ophthalmol. 2014;8: 1507-1513.

10. Escaf LJ, Virgilio Galvis, Alejandro Tello, et al. Ultraphaco and ultraqual techniques using a new ultrasonic Chopper (Ultrachopper). Techniques in Ophthalmology. 2007;5(4):131-1357.

11. Virgilio Galvis, Alejandro tello, Escaf LJ, Victor Rojas, et al. Phaco prechopping as an option in high-volume cataract service. Techniques in Ophthalmology. 2007;5(1):1-7. 